\title{
Improved NN-GM(1,1) for Postgraduates' Employment Confidence Index Forecasting
}

\author{
Lu Wang \\ School of Marxism Studies, Bohai University, Jinzhou 121013, China \\ Correspondence should be addressed to Lu Wang; wanglu@bhu.edu.cn
}

Received 24 May 2014; Revised 30 July 2014; Accepted 1 August 2014; Published 14 August 2014

Academic Editor: Yan-Jun Liu

Copyright (C) $2014 \mathrm{Lu}$ Wang. This is an open access article distributed under the Creative Commons Attribution License, which permits unrestricted use, distribution, and reproduction in any medium, provided the original work is properly cited.

\begin{abstract}
Postgraduates' employment confidence index (ECI) forecasting can help the university to predict the future trend of postgraduates' employment. However, the common forecast method based on the grey model (GM) has unsatisfactory performance to a certain extent. In order to forecast postgraduates' ECI efficiently, this paper discusses a novel hybrid forecast model using limited raw samples. Different from previous work, the residual modified GM(1,1) model is combined with the improved neural network (NN) in this work. In particullar, the hybrid model reduces the residue of the standard GM(1,1) model as well as accelerating the convergence rate of the standard NN. After numerical studies, the illustrative results are provided to demonstrate the forecast performance of the proposed model. In addition, some strategies for improving the postgraduates' employment confidence have been discussed.
\end{abstract}

\section{Introduction}

With the severe situation of social employment, the employment of university students has become more and more difficult. In recent years, the postgraduates are facing stressful employment pressure too. Therefore, the university should pay close attention to both the postgraduates' attitude to the current employment situation and their confidence in the future employment trend [1]. According to the survey, the employment confidence index (ECI) is an indicator designed to measure the confidence of job applicants, which is defined as the degree of optimism on the state of social employment that job applicants are expressing through their attitudes and activities [2,3]. For this reason, the postgraduates' ECI can provide interesting samples to represent their views on employment.

In the past decade, some popular models have been proposed for the ECI forecasting, such as hypothesis testing, linear regression, and grey model (GM). In particular, the GM(1,1) model, based on one variable and first-order derivative, is widely used in academic circles. However, the forecast precision of the model is not satisfying because it operates on the discrete degree of raw sample sequence [4-6]. For this reason, the standard $\mathrm{GM}(1,1)$ model is difficult to forecast the value of future samples accurately [7]. Note that the value of the postgraduates' ECI is often stochastic and nonlinear due to some uncertain factors. How to improve forecasting precision of the postgraduates' ECI has become an important problem. Different from the GM model, the neutral network (NN) is a computational model inspired by the human brain. As an organic system of interconnected neurons, it can calculate the values from multiple inputs simultaneously [811]. Therefore, we can combine the NN with the $\operatorname{GM}(1,1)$ model, called the NN-GM(1,1) model. The hybrid model is a deliberate candidate for effective forecasting. Moreover, the NN can deal with the incomplete and uncertain samples accompanied by the GM-based method. Many researchers made efforts to study the forecast system involving the NNGM $(1,1)$ model, and the relevant work can be found in important literature [12-15]. First, Yang and Chen proposed the intelligent forecast system based on the NN-GM(1,1) model to predict plenty of samples [12]. Bai et al. developed the combined NN-GM(1,1) model for the network traffic forecasting which offered scientific rationale for local area network virus detection, illegal invasion prevention, and Internet routing decision [13]. Then, Ma and Zhu utilized the NN-GM(1,1) model to predict the important samples 
of coal mine safety accidents successfully [14]. Lately, Han et al. established the real-time model predictive control based on the NN-GM(1,1) model for nonlinear systems with prospective accuracy [15]. Meanwhile, the hybrid model cannot get rid of its inherent defects. Both the residue of the $\mathrm{GM}(1,1)$ model and the convergence rate of the $\mathrm{NN}$ still limit forecast performance to a certain degree.

In order to forecast postgraduates' ECI efficiently, it is our intention in this note to study a powerful NN-GM $(1,1)$ model with high forecast precision. To our knowledge, there are few previously published results about it. Then, the contribution of this work can be summarized as follows: (i) the residual modified $\operatorname{GM}(1,1)$ model is presented to reduce residue of the raw sample sequence using differential equation; (ii) the improved $\mathrm{NN}$ is presented to speed up convergence rate using the weights between two adjoining layers; (iii) the improved NN version of the residual modified GM(1,1) model is presented to forecast the postgraduates' ECI.

The remainder of this note is organized as follows. Section 2 addresses the basic principle of the standard GM(1,1) model. In Section 3, the modeling methodology of the improved NN-GM(1,1) model is promoted in detail. In Section 4, the examples are illustrated with efficient results to verify the prediction performance of the proposed model. Moreover, some suggestions on the postgraduates' employment confidence have been discussed. Finally, Section 5 draws the conclusions by presenting the future work.

\section{Preliminaries}

Define the discrete sample sequence as follows:

$$
X^{(0)}=\left(x^{(0)}(1), x^{(0)}(2), \ldots, x^{(0)}(n)\right),
$$

where $n$ is the number of samples [15].

As for the GM(1,1) model, we have the following formula based on the accumulated generating operation (AGO):

$$
x^{(1)}(k)=\sum_{i=1}^{k} x^{(0)}(i), \quad k=1,2, \ldots, n .
$$

Then, the incremental sample sequence is

$$
X^{(1)}=\left(x^{(1)}(1), x^{(1)}(2), \ldots, x^{(1)}(k)\right) .
$$

The differential equation of $x^{(1)}(k)$ is

$$
\dot{x}^{(1)}(k)+a x^{(1)}(k)=u,
$$

where $a$ is the development coefficient and $u$ is the grey input of the $\operatorname{GM}(1,1)$ model $[16,17]$. According to the least square law, we have

$$
\left[\begin{array}{l}
a \\
u
\end{array}\right]=\left(B_{1}^{\mathrm{T}} B_{1}\right)^{-1} B_{1}^{\mathrm{T}} Y_{1}
$$

where $(\cdot)^{\mathrm{T}}$ denotes the transpose of matrix; $Y_{1}$ and $B_{1}$ are given by

$$
\begin{aligned}
& Y_{1}=\left[\begin{array}{llll}
x^{(0)}(2) & x^{(0)}(3) & \cdots & x^{(0)}(n)
\end{array}\right]^{\mathrm{T}} \\
& B_{1}=\left[\begin{array}{cc}
-\frac{1}{2}\left(x^{(1)}(1)+x^{(1)}(2)\right) & 1 \\
-\frac{1}{2}\left(x^{(1)}(2)+x^{(1)}(3)\right) & 1 \\
\vdots & \vdots \\
-\frac{1}{2}\left(x^{(1)}(n-1)+x^{(1)}(n)\right) & 1
\end{array}\right] .
\end{aligned}
$$

Then, the grey predicted formula is

$$
\widehat{x}^{(1)}(k+1)=\left(x^{(0)}(1)-\frac{u}{a}\right) e^{-a k}+\frac{u}{a} .
$$

Furthermore, the predicted sample can be obtained by the first-order differential equation. That is,

$$
\begin{aligned}
\widehat{x}^{(0)}(k+1) & =\widehat{x}^{(1)}(k+1)-\widehat{x}^{(1)}(k) \\
& =\left(1-e^{a}\right) e^{-a k}\left(x^{(0)}(1)-\frac{u}{a}\right) .
\end{aligned}
$$

Remark 1. Compared with other forecast models, the GM(1,1) model need not find statistical features of the raw samples. It overcomes the shadow of sample statistics using sample sequence $[7,18,19]$. However, we note that the limited number of the raw samples in the set $X^{(0)}$ would bring about the residue. It is likely to affect the forecast precision and, therefore, cause the incorrect decision.

\section{Methodology}

Note that the forecast precision of the standard $\operatorname{GM}(1,1)$ model is unsatisfactory. We derive an improved $\mathrm{NN}$ version of the residual modified GM(1,1) model in this section.

3.1. Residual Modified GM(1,1) Model. We have in hand the residue of $x^{(1)}(k)$ as $[20,21]$

$$
\begin{aligned}
e^{(1)}(k) & =\sum_{i=1}^{k} e^{(0)}(i) \\
& =\sum_{i=1}^{k}\left[x^{(0)}(i)-\widehat{x}^{(0)}(i)\right] .
\end{aligned}
$$

Similar to (7), the predicted residue can be written as

$$
\hat{e}^{(1)}(k+1)=\left(e^{(0)}(1)-\frac{u}{a}\right) e^{-a k}+\frac{u}{a} .
$$

Then, the differential equation of (10) is

$$
\dot{\hat{e}}^{(1)}(k+1)=-a\left(e^{(0)}(1)-\frac{u}{a}\right) e^{-a k},
$$

where $\dot{\hat{e}}^{(1)}(k+1)$ represents the change rate of two neighbouring samples. 
Next, we update (7) using the following formula:

$$
\begin{aligned}
\widehat{x}^{(1)}(k+1) & =\widehat{x}^{(1)}(k+1)+\dot{\hat{e}}^{(1)}(k+1) \\
& =\left(x^{(0)}(1)-\frac{u}{a}\right) e^{-a k}+\frac{u}{a}-a\left(e^{(0)}(1)-\frac{u}{a}\right) e^{-a k} .
\end{aligned}
$$

At time $k+1$, the modified predicted sample can be obtained as

$$
\begin{aligned}
\widehat{x}^{(0)}(k+1)= & \widehat{x}^{(1)}(k+1)-\widehat{x}^{(1)}(k) \\
= & \left(1-e^{a}\right) e^{-a k} \\
& \times\left(\left(x^{(0)}(1)-\frac{u}{a}\right)-a\left(e^{(0)}(1)-\frac{u}{a}\right)\right) \\
= & \widehat{x}^{(0)}(k+1)+\left(1-e^{a}\right) \dot{\hat{e}}^{(1)}(k+1) .
\end{aligned}
$$

Remark 2. Considering the differential of the predicted residue, $\widehat{x}^{(0)}(k+1)$ is smoother than $\widehat{x}^{(0)}(k+1)$ using the residual modified GM(1,1) model.

3.2. Principle of NN. As we know, the $\mathrm{NN}$ is modeled from the physical architecture of the human brain. With flexible property and learning ability, it can be used to model the unknown relationship between inputs and outputs. As it makes further prediction based on the outcomes from the GM(1,1) model and takes advantage of the orderliness and boundedness of samples, the NN can make the predicted results more precise in addition to the errors controllable [2224].

Considering a 4-layer $\mathrm{NN}$, the input layer has $m_{1}$ neurons, two hidden layers have $m_{2}$ and $m_{3}$ neurons, and the output layer has $m_{4}$ neurons. Assume that $w_{s t}$ and $\theta_{t}$ are the weight and the threshold from the input layer to the first hidden layer; $w_{t r}^{\prime}$ and $\theta_{r}^{\prime}$ are the weight and the threshold between two hidden layers; and $w_{r l}^{\prime \prime}$ and $\theta_{l}^{\prime \prime}$ are the weight and the threshold from the second hidden layer to the output layer; then the neurons of the input layer, the first hidden layer, the second hidden layer, and the output layer can be defined as follows:

$$
\begin{gathered}
x_{s}=\left(x_{1}, x_{2}, \ldots, x_{m_{1}}\right)^{\mathrm{T}}, \\
x_{t}^{\prime}=f\left(\sum_{s=0}^{m_{2}-1} w_{s t} x_{s}-\theta_{t}\right)^{\mathrm{T}}, \\
x_{r}^{\prime \prime}=f\left(\sum_{t=0}^{m_{3}-1} w_{t r}^{\prime} x^{\prime}-\theta_{r}^{\prime}\right)^{\mathrm{T}}, \\
y_{l}=f\left(\sum_{r=0}^{m_{4}-1} w_{r l}^{\prime \prime} x_{r}^{\prime \prime}-\theta_{l}^{\prime \prime}\right)^{\mathrm{T}},
\end{gathered}
$$

where $f(\cdot)$ denotes the Tansig function; that is,

$$
f(u)=\frac{1}{1+e^{-u}}
$$

Furthermore, the differential of (15) is given by

$$
\begin{aligned}
\frac{\mathrm{d} f(u)}{\mathrm{d} u} & =\frac{e^{-u}}{\left(1+e^{-u}\right)^{2}} \\
& =f(u)(1-f(u)) .
\end{aligned}
$$

Assume that $y_{l}^{(p)}$ and $d_{l}^{(p)}$ are the predicted value and the desired value of thepth output; then the quadratic error is given by

$$
E^{(p)}=\frac{1}{2} \sum_{l=0}^{m_{4}-1}\left(d_{l}^{(p)}-y_{l}^{(p)}\right)^{2} .
$$

Remark 3. Owing to the dynamic changes such as the huge number of samples and parameters to be learned and trained, the standard NN cannot be guaranteed to rapidly converge when it is far away from a local minimum [25-27]. Consequently, its convergence rate is unsuitable for practical applications.

3.3. Improved NN. We present an improved NN to speed up the convergence rate based on the batch-correction method. Starting with (15), the total errors are defined as

$$
\begin{aligned}
E_{A} & =\sum_{p=1}^{P} E^{(p)} \\
& =\frac{1}{2} \sum_{p=1}^{P} \sum_{l=0}^{m_{4}-1}\left(d_{l}^{(p)}-y_{l}^{(p)}\right)^{2} .
\end{aligned}
$$

Assume that $\eta$ is the learning rate and $\tau$ is the iteration number; the gradient of $E_{A}$ is given by

$$
\nabla=-\eta \frac{\partial E_{A}}{\partial w(\tau)} .
$$

Then, the modified weight of the output layer is

$$
\begin{aligned}
w_{r l}^{\prime \prime}(\tau+1) & =w_{r l}^{\prime \prime}(\tau)+\nabla^{\prime \prime} \\
& =w_{r l}^{\prime \prime}(\tau)-\eta \frac{\partial E_{A}}{\partial w_{r l}^{\prime \prime}(\tau)} \\
& =w_{r l}^{\prime \prime}(\tau)-\eta \sum_{p=1}^{P} \frac{\partial E^{(p)}}{\partial y_{l}^{(p)}} \frac{\partial y_{l}^{(p)}}{\partial u_{l}^{\prime \prime(p)}} \frac{\partial u_{l}^{\prime \prime}(p)}{\partial w_{r l}^{\prime \prime}} \\
& =w_{r l}^{\prime \prime}(\tau)+\eta \sum_{p=1}^{P}\left(d_{l}^{(p)}-y_{l}^{(p)}\right) y_{l}^{(p)}\left(1-y_{l}^{(p)}\right) x_{r}^{\prime \prime(p)} \\
& =w_{r l}^{\prime \prime}(\tau)+\eta \sum_{p=1}^{P} \delta_{r l}^{(p)} x_{r}^{\prime \prime(p)}
\end{aligned}
$$

where $y_{l}^{(p)}=f\left(u_{l}^{\prime \prime(p)}\right)^{T}, u_{l}^{\prime \prime(p)}=\sum_{r=0}^{m_{4}-1} w_{r l}^{\prime \prime} x_{r}^{\prime \prime(p)}-\theta_{l}^{\prime \prime}$, and $\delta_{r l}^{(p)}=\left(d_{l}^{(p)}-y_{l}^{(p)}\right) y_{l}^{(p)}\left(1-y_{l}^{(p)}\right)$. 
TABLE 1: Raw samples.

\begin{tabular}{lccccc}
\hline Year & Male & Female & Science & Social science & Global \\
\hline 2006 & 146 & 139 & 143 & 130 & 127 \\
2007 & 140 & 133 & 138 & 118 & 137 \\
2008 & 137 & 127 & 131 & 110 & 129 \\
2009 & 128 & 116 & 123 & 122 & 120 \\
2010 & 139 & 130 & 135 & 113 & 133 \\
2011 & 130 & 120 & 128 & 107 & 125 \\
2012 & 125 & 112 & 117 & 105 & 114 \\
2013 & 123 & 109 & & \\
\hline
\end{tabular}

The modified weight of the second hidden layer is

$$
\begin{aligned}
w_{t r}^{\prime}(\tau+1) & =w_{t r}^{\prime}(\tau)+\nabla^{\prime} \\
& =w_{t r}^{\prime}(\tau)-\eta \frac{\partial E_{A}}{\partial w_{t r}^{\prime}(\tau)} \\
& =w_{t r}^{\prime}(\tau)-\eta \sum_{p=1}^{P} \frac{\partial E^{(p)}}{\partial y_{l}^{(p)}} \frac{\partial y_{l}^{(p)}}{\partial u_{l}^{\prime \prime(p)}} \frac{\partial u_{l}^{\prime \prime(p)}}{\partial x_{r}^{\prime \prime(p)}} \frac{\partial x_{r}^{\prime \prime}}{\partial u_{r}^{\prime(p)}} \frac{\partial u_{r}^{\prime(p)}}{\partial w_{t r}^{\prime}} \\
& =w_{t r}^{\prime}(\tau)+\eta \sum_{p=1}^{P}\left(\begin{array}{l}
\left(d_{l}^{(p)}-y_{l}^{(p)}\right) y_{l}^{(p)}\left(1-w_{r l}^{\prime \prime} x_{r}^{\prime \prime(p)}\left(1-x_{r}^{\prime \prime(p)}\right) x_{t}^{\prime(p)}\right) \\
\end{array}\right. \\
& =w_{t r}^{\prime}(\tau)+\eta \sum_{p=1}^{P} \delta_{t r}^{(p)} x_{t}^{\prime(p)},
\end{aligned}
$$

where $u_{r}^{\prime(p)}=\sum_{t=0}^{m_{3}-1} w_{t r}^{\prime} x_{t}^{\prime(p)}-\theta_{r}^{\prime}$ and $\delta_{t r}^{(p)}=\delta_{r l}^{(p)} w_{r l}^{\prime \prime} x_{r}^{\prime \prime(p)}(1-$ $\left.x_{r}^{\prime \prime(p)}\right)$.

Similarly, the modified weight of the first hidden layer is

$$
w_{s t}(\tau+1)=w_{s t}(\tau)+\eta \sum_{p=1}^{P} \delta_{s t}^{(p)} x_{s}^{(p)}
$$

where $\delta_{s t}^{(p)}=\delta_{t r}^{(p)} w_{t r}^{\prime} x_{t}^{\prime(p)}\left(1-x_{t}^{\prime(p)}\right)$.

Subsequently, we introduce the momentum item $\alpha(w(\tau-$ 1) $-w(\tau-2))$ to represent (20)-(22) using the uniform formula

$$
w(\tau+1)=w(\tau)+\nabla+\alpha(w(\tau-1)-w(\tau-2)),
$$

where $\alpha$ denotes the momentum factor.

Remark 4. Given that the directions of $\nabla$ and $\alpha(w(\tau-1)-$ $w(\tau-2))$ are opposite, the sum of them plays a positive role in the yields of $w(\tau+1)$. In other words, the modified weight can be dynamically adjusted on the basis of the current gradient and the previous momentum at each cycle in the training stage $[28,29]$. In addition, the error sensitivity of the modified weight is reduced to a local minimum state. Accordingly, the convergence rate of the proposed NN is rapidly improved.

3.4. Implementation of Improved NN-GM(1,1) Model. We investigate the postgraduates' ECI of a certain university from 2006 to 2013 . The raw samples are given in Table 1 that

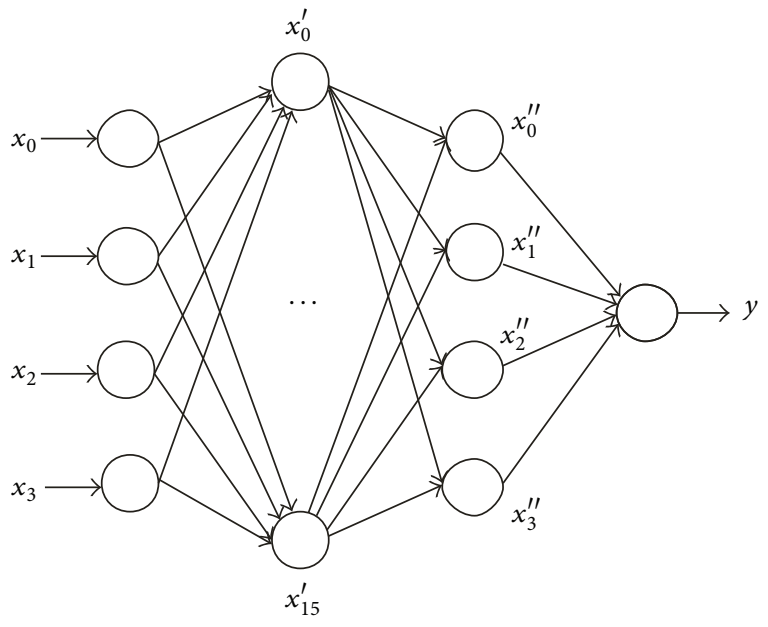

FIGURE 1: Topology structure of NN.

contains four different categories such as the male students' ECI, female students' ECI, science students' ECI, and social science students' ECI. Besides all, the global ECI is also shown in this table. As we know, the value of the ECI is in the interval $[0,200]$. In particular, the minimum value 0 denotes the no confidence; the maximum value 200 denotes the great confidence; and the median value 100 means the neutral attitude. When the value of the ECI is more than 100, the confidence is positive. On the contrary, when the value of the ECI is less than 100, the confidence is negative.

In Table 1, we note that the values of the global ECI and four kinds of ECIs are nonlinear. Compared with the female students, the male students have lager value; compared with the social science students, the science students have the lager value. In addition, the values of five kinds of ECIs are down from 2006 to 2013 as a whole. In particular, the value reaches the minimum in 2013 because the number of postgraduates has exploded.

To forecast the global ECI this year, we consider the improved model in Figure 1. The input layer has 4 nodes, two hidden layers have 16 and 4 nodes, and the output layer has 1 node. In the topology structure, every four inputs are corresponding to one output, which means that the global ECI is determined by four individual ECIs.

Next, we employ the residual modified GM $(1,1)$ model to smooth the raw samples and then obtain the corrected results 
TABLE 2: Corrected results on residual modified GM(1,1) model.

\begin{tabular}{lcccc}
\hline Year & Male & Female & Science & Social science \\
\hline 2006 & 146.00 & 139.00 & 143.00 & 130.00 \\
2007 & 140.00 & 133.00 & 138.00 & 127.00 \\
2008 & 140.55 & 122.69 & 127.93 & 115.07 \\
2009 & 130.91 & 119.54 & 125.62 & 112.86 \\
2010 & 134.62 & 120.79 & 131.58 & 118.24 \\
2011 & 131.78 & 117.37 & 128.40 & 114.59 \\
2012 & 124.03 & 114.04 & 118.74 & 105.85 \\
2013 & 121.79 & 110.81 & 116.47 & 103.46 \\
2014 & 119.58 & 104.69 & 114.21 & 101.07 \\
\hline
\end{tabular}

in Table 2. It is obvious that the residual modified $\operatorname{GM}(1,1)$ model keeps the first two values unchanged and predicts four individual ECIs in 2014.

For comparison, Figure 2 shows the difference of two tables. In this figure, it can be seen that the residual modified $\mathrm{GM}(1,1)$ model has smaller value of the root mean square error (RMSE), which means the values of ECIs in Table 2 are smoother.

Finally, we extract the values of four individual ECIs from Table 2 every year for each group as inputs and draw the corresponding global ECI from Table 1 as the desired output. After learning and training, the global ECI in 2014 can be obtained.

\section{Numerical Studies and Discussions}

This section presents the numerical studies to compare prediction performance of the standard GM(1,1) model and that of the proposed model under MATLAB v7.8.

First, the proposed NN is kept working when the value of error reaches the preset value $10^{-5}$ based on the samples in Tables 1 and 2. Figures 3 and 4 demonstrate the learning performance and the training performance of the improved $\mathrm{NN}$, respectively. As shown in Figure 3, both the gradient and the learning rate can be obtained in the whole training processing. At the 8069th epoch, the values of the gradient and the learning rate are $7.5912 \times 10^{-5}$ and 0.8421 , which means that the proposed $\mathrm{NN}$ has the best learning performance at this epoch.

From Figure 4, it can be seen that the value of the mean square error (MSE) is decreasing from $10^{-1}$ to $10^{-5}$. In particular, the value of MSE reaches the boundary value $10^{-5}$ at the 8069th epoch, which means that the proposed NN obtains the best training performance at this epoch.

After learning and training, the forecast results based on the standard GM(1,1) model given [3], the proposed model, and the original series are plotted in Figure 5, respectively. From this figure, it is clear that the trend of the postgraduates' ECI can be forecasted based on the standard $\operatorname{GM}(1,1)$ model. However, the forecast precision of this model is unstable at some points. The reason can be explained that the accumulated sequence obtained using the AGO formation is monotonically increasing, and, therefore, the standard

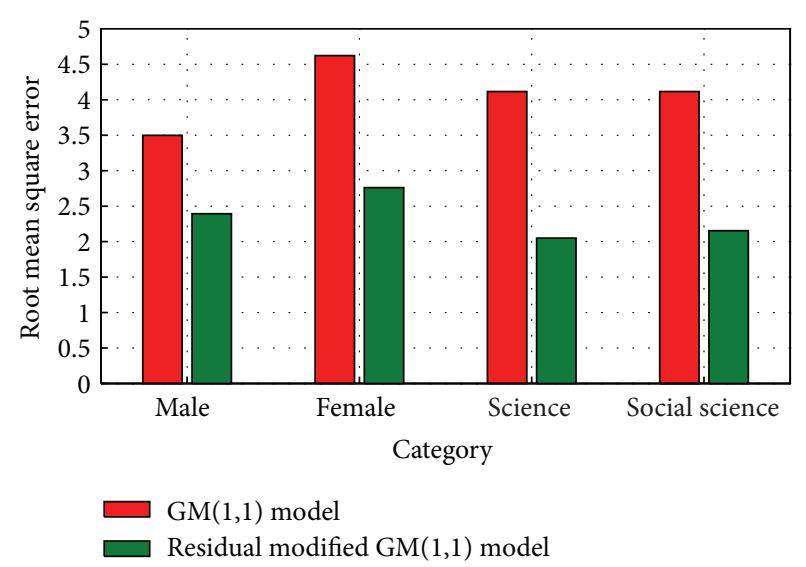

FIGURE 2: Comparison of RMSE.

GM(1,1) model cannot effectively extract the random fluctuations of the raw samples. As a result, the postgraduates' ECI forecasting using this model is far from satisfactory. That is, as a declining curve, it deviates from the raw samples. By comparison, the predicted values of ECIs using the proposed model are approaching the raw samples, where the four values in 2006, 2007, 2009, and 2013 are almost equivalent.

Figure 6 depicts the relative error comparison of two models. In this figure, it is obvious that the proposed model has only $28.7 \%$ relative error of the standard GM(1,1) model. Therefore, the value of the global ECI 114.63 in 2014 using the proposed model in Figure 5 is more credible. Compared with the value of the postgraduates' ECI in 2013, this value is a little larger.

Remark 5. On the basis of numerical results, we note that the proposed model forecasts the global ECI this year with prospective precision at the expense of computational load. As the residual modified $\mathrm{GM}(1,1)$ model and the improved $\mathrm{NN}$ are utilized in the proposed model, the computational complexity of the proposed model is slightly higher. With respect to the reliability, the model is more competent. In addition, we also find that the postgraduates' employment is a problem about structure and system but not an overall problem [30]. Admittedly, the improvement of the postgraduates' employment confidence involves the students' activities; that is, they should do their utmost to study hard, widen 


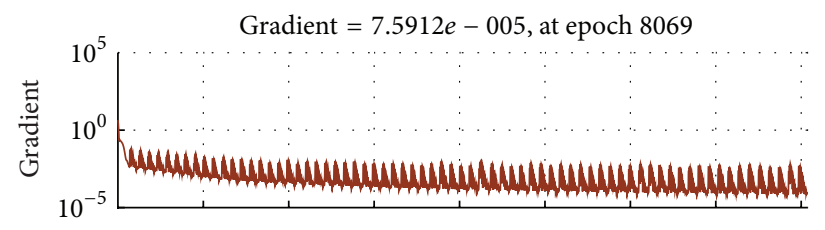

Learning rate $=0.84209$, at epoch 8069

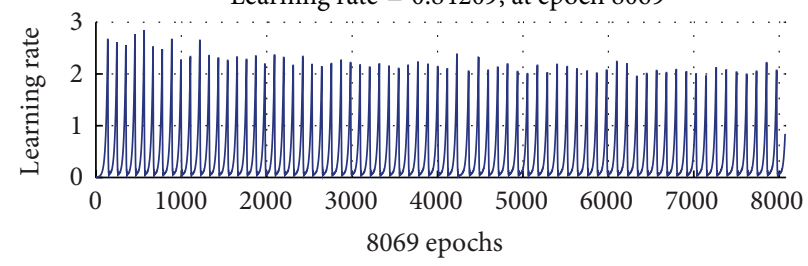

Figure 3: Gradient and learning rate.

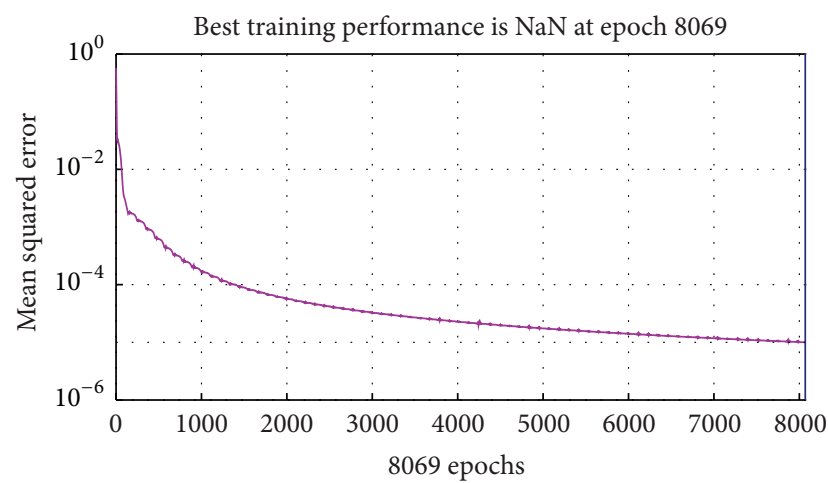

Figure 4: Mean square error.

knowledge, and improve creative ability. Furthermore, it is a comprehensive matter related to the government, university, and employer, which should establish scientific concepts of talents and constant fair competition systems as well as taking steps to provide more employment opportunities. Together, the relationship among the government, university, employer, and postgraduates would be well balanced in order to take the following measures to improve the postgraduates' employment confidence [2, 3, 31, 32]: (i) improve the postgraduates' qualities and employment ability based on the career life planning during the graduate level; (ii) cultivate the postgraduates' viewpoints of employment and enterprising spirits so as to accumulate the experience on seeking job and achieve employment-oriented goal; (iii) encourage the postgraduates to work in grassroots workplaces in rural towns and city streets, where the students will be provided with more careers in management and public service; (iv) advise the postgraduates to work in small and medium enterprises and nonpublic enterprises, where the students will face more challenges and gain more knowledge; and (v) guide the postgraduates to start their own business, such as the flexible employment based on national entrepreneurship policy.

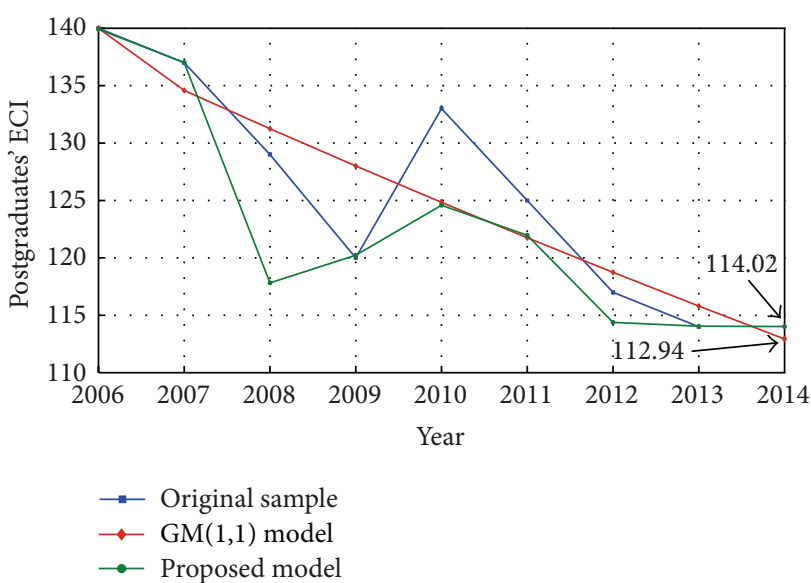

Figure 5: Comparison of ECI.

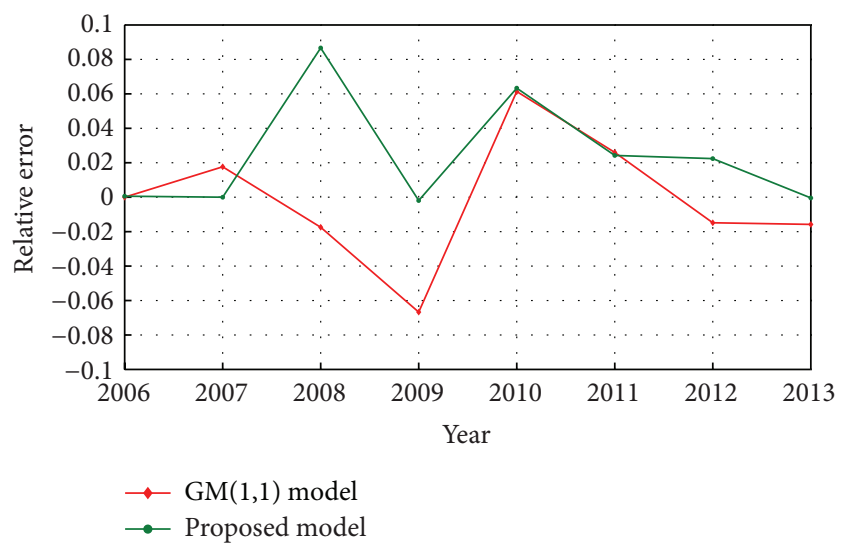

FIGURE 6: Comparison of relative error.

\section{Conclusions}

This paper presents an improved NN-GM(1,1) model for the postgraduates' ECI forecasting. Combining the residual modified $\operatorname{GM}(1,1)$ with the improved $\mathrm{NN}$, we reduce the residue of the GM(1,1) model as well as improving the convergence rate of the NN. By taking advantage of the hybrid model, we successfully forecast the global ECI in 2014 with the last eight years' samples. We can conclude that the postgraduates' ECI this year is relatively optimistic. In addition, some strategies for improving the postgraduates' employment confidence have been discussed. Consequently, this work has provided a valuable reference to the postgraduates' ECI forecasting using limited samples. With the help of the increasing samples in the future, we believe that the application of the proposed model would get higher accuracy. In the future research, how to reduce the computational complexity will be further focused on.

\section{Conflict of Interests}

The author declared that there is no conflict of interests regarding the publication of this paper. 


\section{Acknowledgments}

This work is partially supported by the National Natural Science Foundation of China (no. 61104017) and the Subject of the Education \& Science Planning Introduction of the "12th Five-Year," Liaoning Province (no. JG12DB280). The author gratefully acknowledges the helpful suggestions of Dr. Bo Li.

\section{References}

[1] P. Dai, Mathematical model of the employment population and prediction [M.S. thesis], Zhejiang University, Hangzhou, China, 2010.

[2] C. H. Yan, The design and analysis of employment confidence index of college student [M.S. thesis], Zhejiang Normal University, Hangzhou, China, 2007.

[3] G. J. Yang, "College students' employment confidence index prediction based on GM(1,1)," Light Industry Science and Technology, vol. 6, pp. 174-175, 2013.

[4] Y. H. Wang, Q. Liu, J. R. Tang, W. B. Cao, and X. Z. Li, "Optimization approach of background value and initial item for improving prediction precision of GM(1,1) model," Journal of Systems Engineering and Electronics, vol. 25, no. 1, pp. 77-82, 2014.

[5] X. P. Xiao and J. L. Deng, "A new modified GM (1,1) model: grey optimization model," Journal of Systems Engineering and Electronics, vol. 12, no. 2, pp. 1-5, 2001.

[6] E. Kayacan, B. Ulutas, and O. Kaynak, "Grey system theorybased models in time series prediction," Expert Systems with Applications, vol. 37, no. 2, pp. 1784-1789, 2010.

[7] N. B. Zhao, J. L. Yang, S. Y. Li, and Y.-W. Sun, "Markov chainbased aeroengine performance degradation forecast approach using exhaust gas temperature," Mathematical Problems in Engineering, vol. 2014, Article ID 832851, 11 pages, 2014.

[8] W. Yan, "Toward automatic time-series forecasting using neural networks," IEEE Transactions on Neural Networks and Learning Systems, vol. 23, no. 7, pp. 1028-1039, 2012.

[9] Y. J. Liu, L. Tang, S. C. Tong, and C. L. P. Chen, "Adaptive NN controller design for a class of nonlinear MIMO discrete-time systems," IEEE Transactions on Neural Networks and Learning Systems, no. 99, 1 page, 2014.

[10] D. Z. Li, W. Wang, and F. Ismail, "Fuzzy neural network technique for system state forecasting," IEEE Transactions on Cybernetics, vol. 43, no. 5, pp. 1484-1494, 2013.

[11] Y. Liu, L. Liu, and S. Tong, "Adaptive neural network tracking design for a class of uncertain nonlinear discrete-time systems with dead-zone," Science China Information Sciences, vol. 57, no. 3, pp. 1-12, 2014.

[12] S. H. Yang and Y. P. Chen, "Intelligent forecasting system based on grey model and neural network," in Proceedings of the IEEE/ASME International Conference on Advanced Intelligent Mechatronics (AIM '09), pp. 699-704, Singapore, July 2009.

[13] Y. Bai, K. Ma, and Q. C. Ren, "A NN-GM (1,1) model-based analysis of network traffic forecasting," in Proceedings of the 3rd IEEE International Conference on Broadband Network and Multimedia Technology (IC-BNMT '10), pp. 191-194, Beijing, China, October 2010.

[14] H. Ma and Y. Zhu, "Forecast and analysis of coal mine safety accidents based on BP Neural Network and GM Model," in Proceedings of the 2nd IEEE International Conference on Emergency Management and Management Sciences (ICEMMS '11), pp. 109-111, Beijing, China, August 2011.
[15] H. G. Han, X. L. Wu, and J. F. Qiao, "Real-time model predictive control using a self-organizing neural network," IEEE Transactions on Neural Networks and Learning Systems, vol. 24, no. 9, pp. 1425-1436, 2013.

[16] Z. Jin, "The application of BP neural network and gray neural network in university graduate's employment forecasting," in Proceedings of the 3rd International Conference on Advanced Computer Theory and Engineering (ICACTE '10), pp. V2522V2526, Chengdu, China, August 2010.

[17] H. D. Long and G. L. Yan, "Research on GDP time series forecasting based on integrating SARIMA, GM(1,1) and BP neural networks," Journal of Applied Statistics and Management, vol. 32, no. 5, pp. 814-822, 2009.

[18] S. F. Liu, B. Zhang, J. F. Liu, and N. M. Xie, "Several basic models of $\operatorname{GM}(1,1)$ and their applicable bound," Systems Engineering and Electronics, vol. 36, no. 3, pp. 77-82, 2014.

[19] K. Zhang, S. Mao, and W. Yuan, "Appling modification grey model based on Markov-chain to highway network planning," Journal of Wuhan University of Technology, vol. 29, no. 4, pp. 503-505, 2005.

[20] Y. X. Gao, J. G. Gao, Z. P. Wang, Q. H. Li, Y. Z. Qiu, and H. L. Chen, "The passenger traffic volume prediction system of GM $(1,1)$ model algorithm," Electronic Design Engineering, vol. 22, no. 7, pp. 16-18, 2014.

[21] G. Li, S. Masuda, and M. Nagai, "The prediction model for electrical power system using an improved hybrid optimization model," International Journal of Electrical Power \& Energy Systems, vol. 44, no. 1, pp. 981-987, 2013.

[22] H. S. Shi, A. W. Fan, and W. Wang, "Prediction of coal demand the based on Markvo residual modification and grey theory," Computer Simulation, vol. 28, no. 10, pp. 187-190, 2011.

[23] Y. Liu, C. L. P. Chen, G. Wen, and S. Tong, "Adaptive neural output feedback tracking control for a class of uncertain discrete-time nonlinear systems," IEEE Transactions on Neural Networks, vol. 22, no. 7, pp. 1162-1167, 2011.

[24] H. Wang and H. Q. Cheng, "Modified blind equalization algorithm based on back-propagation neural networks with adaptive momentum factor," Computer Engineering and Design, vol. 31, no. 6, pp. 1297-1300, 2005.

[25] Y. Liu, S. Tong, D. Wang, T. Li, and C. L. P. Chen, "Adaptive neural output feedback controller design with reduced-order observer for a class of uncertain nonlinear SISO Systems," IEEE Transactions on Neural Networks, vol. 22, no. 8, pp. 1328-1334, 2011.

[26] J. Wang, J. Yang, and W. Wu, "Convergence of cyclic and almost-cyclic learning with momentum for feedforward neural networks," IEEE Transactions on Neural Networks, vol. 22, no. 8, pp. 1297-1306, 2011.

[27] Y.-J. Liu and S. C. Tong, "Adaptive neural network tracking control of uncertain nonlinear discrete-time systems with nonaffine dead-zone input," IEEE Transactions on Cybernetics, no. 99, 1 page, 2014.

[28] H. Wang, "Modified blind equalization algorithm based on back-propagation neural networks with adaptive momentum factor," Computer Engineering and Design, vol. 31, no. 6, pp. $1297-1300,2010$

[29] J. X. Yang, N. Liu, and Y. C. Liu, "Risk evaluation of PFI project based on momentum BP neural network," Journal of Shenyang Jianzhu University, vol. 15, no. 2, pp. 202-204, 2013.

[30] S. Q. Li, H. Li, and L. L. Jin, "The analysis of college students employment stressors," Tsinghua Journal of Education, vol. 32, no. 2, pp. 71-76, 2011. 
[31] H. X. Zhou, "Investigation and countermeasures on employment confidence of college students," Journal of Northeast Normal University, vol. 251, no. 3, pp. 214-217, 2011.

[32] C. Q. Cheng, Research on issues of employment of college Students in China [M.S. thesis], Wuhan University, Wuhan, China, 2010. 


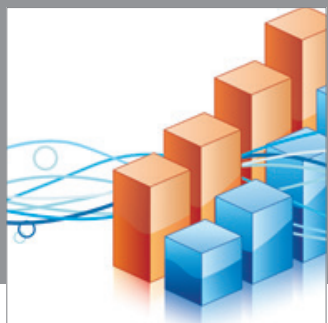

Advances in

Operations Research

mansans

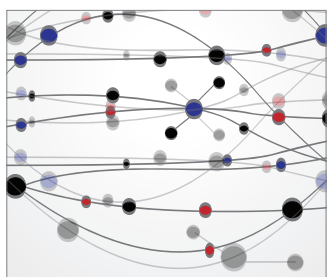

The Scientific World Journal
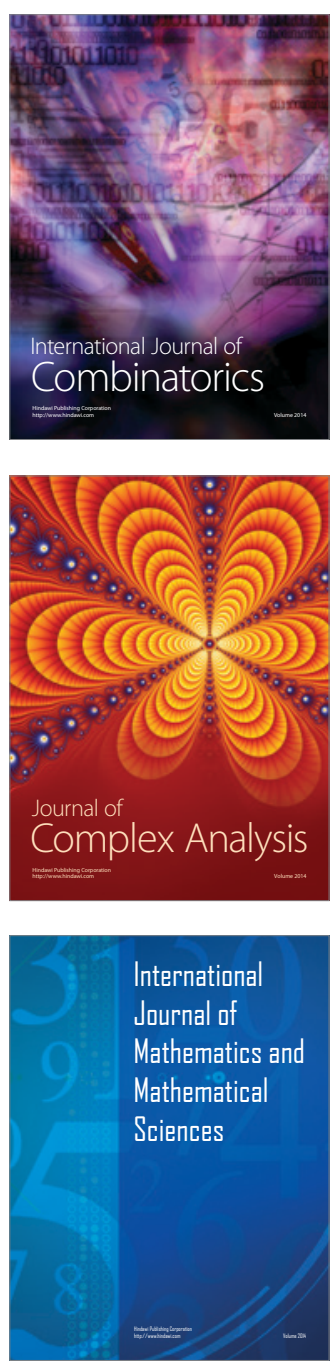
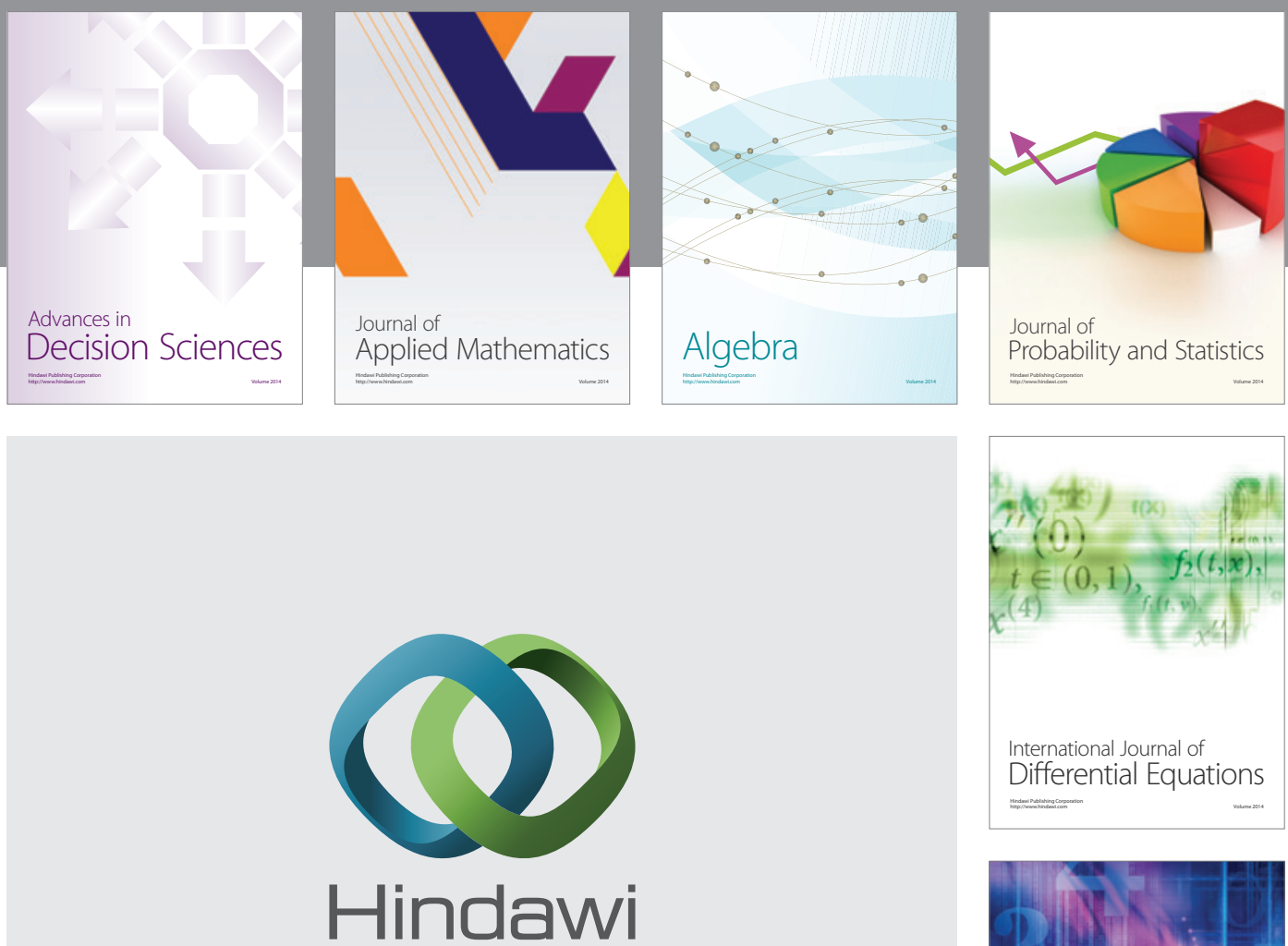

Submit your manuscripts at http://www.hindawi.com
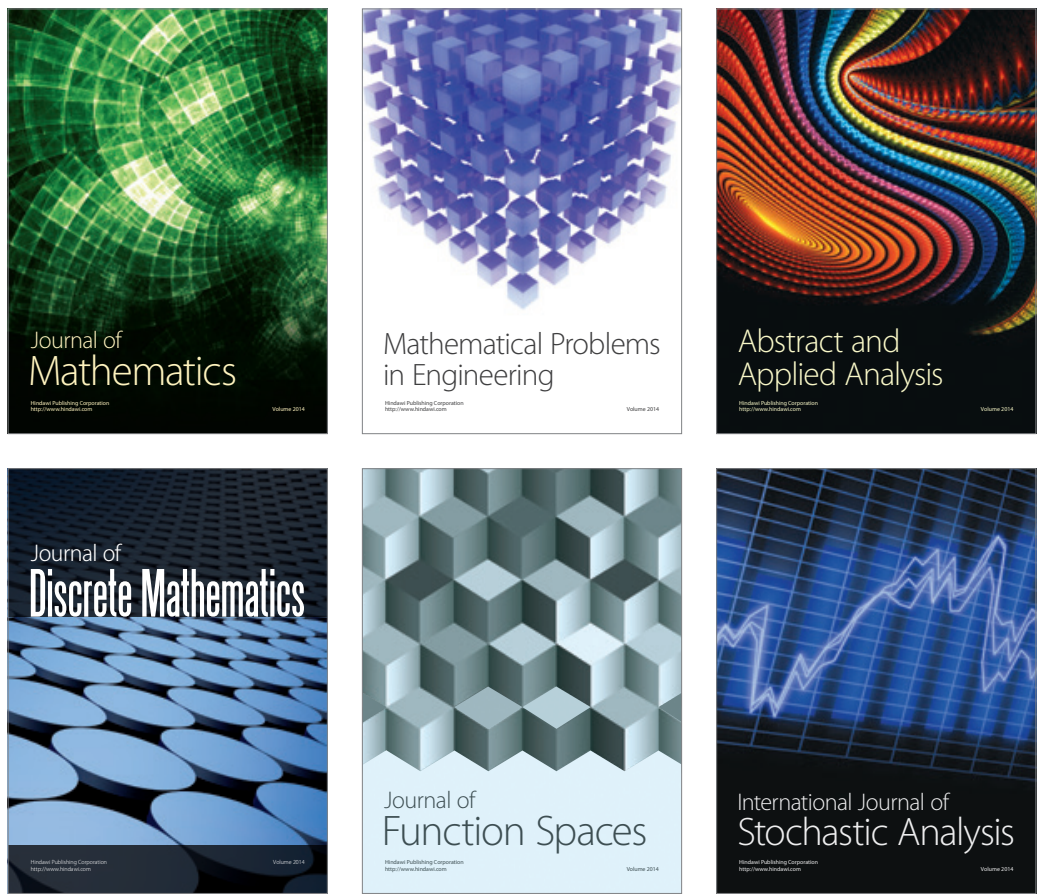

Journal of

Function Spaces

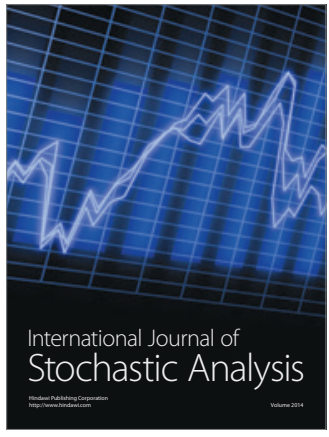

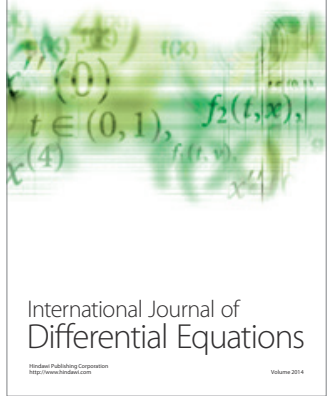
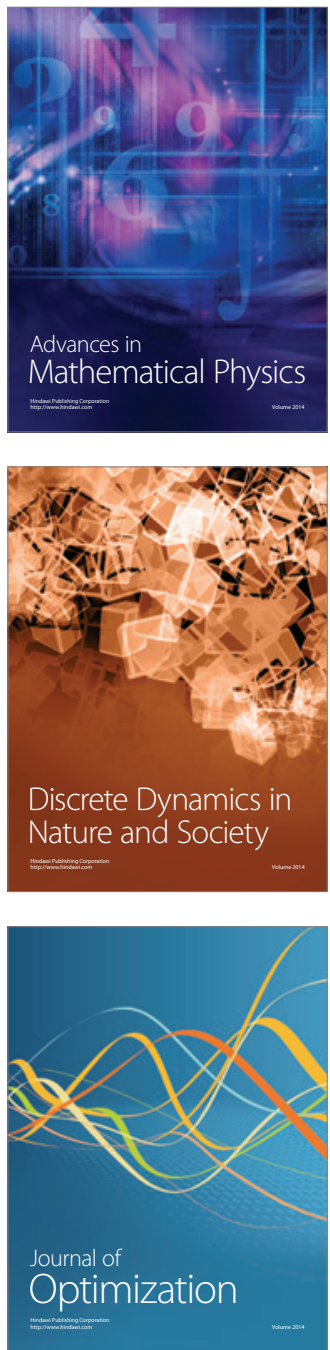